transfection on cell activity and migration in unrestricted space and through pores are larger than nucleus. The presence of various lamin A isoforms in NL is likely to lead to a reduction of migration ability due to the increase of the nuclear rigidity. The absence of effect of lamin A knockdown on migration may be explained that HT1080 cell line is supposed to have initially high migration ability and, possibly, more elastic NL and, consequently, it has originally reduced lamin A level. Therefore our next target is to estimate the level of lamin A isoforms by RT-PCR and WB and to conduct similar experiments by using other cell lines.

This work was supported by Russian Science Fund (RSF) № 17-15-01290.

doi: http://dx.doi.org/10.7124/bc.0009F3

\section{P-1. Electron microscopic approaches in studies of lipidic structures in the cell nucleus}

\author{
Vlada Philimonenko ${ }^{1,2}$, Zuzana Dolejšíi ${ }^{2}$, \\ Margarita Sobol ${ }^{1}$, Dominik Pinkas ${ }^{2}$, Toyo- \\ shi Fujimoto ${ }^{3}$, Pavel Hozák ${ }^{1}$ \\ ${ }^{1}$ Department of Biology of the Cell Nucleus, Insti- \\ tute of Molecular Genetics ASCR, Prague, Czech \\ Republic; ${ }^{2}$ Microscopy Centre, Electron Micros- \\ copy Core Facility, Institute of Molecular Genetics \\ ASCR, Prague, Czech Republic; ${ }^{3}$ Department of \\ Molecular Cell Biology, Nagoya University Gradu- \\ ate School of Medicine, Nagoya, Japan \\ vlada@img.cas.cz
}

So far, mostly protein complexes have been found as important for this ordering. We have described novel structures containing phosphatidylinositol 4,5-bisphosphate (PIP2) which seem to contribute as well. Ultrastructural studies demonstrate the PIP2-positive structures propagating through the nucleolus into the nucleoplasm where PIP2 is enriched in interchromatin granules, and also form previously undescribed 70-100 nm roundish "nuclear lipid islets" (NLI). Electron microscopy (EM) is essential for thorough characterization of the structure and molecular composition of this novel nuclear compartment. The usage of appropriate method for EM sample preparation is a crucial step as lipidic structures are potentially predisposed to extraction or translocations. Aims: To verify the results of ultrastructural localization of PIP2 obtained by several approaches including chemical fixation and cryoimmobilization followed by different immunolabeling techniques. Methods: We employed quick-freezing - freeze fracture replica labelling (QF-FRL), where the biological material is fixed physically - by high-pressure freezing, and subsequently mechanically - by coating with carbon/platinum layer. Due to it, QFFRL fixes also lipids and the samples are considered to be closer to the native state as compared to other techniques. Results: We were able to immunolabel PIP2 present in membranes as well as PIP2 which is localized in non-membranous compartments. NLI in the nucleoplasm were of the same size and shape as revealed by other techniques. Conclusions: The results demonstrate that NLI are stable structures resistant to aldehyde fixation and extraction, which is possibly ensured by interactions of lipidic components with proteins.

GACR (15-08738S, 19-05608S), TACR (TE01020118), CAS (JSPS-18-18), institutional grant (RVO: 68378050), MEYS CR (LM2015062), OPVVV (CZ.02.1.01/0.0/16 013/0001775). 